\title{
Resultados maternos y perinatales de preeclampsia en el Hospital Hipólito Unanue de Tacna
}

\section{Maternal and perinatal results of preeclampsia Hipolito Unanue Hospital in Tacna}

\section{Angela Farfán Zaga ${ }^{\text {1a }}$ \\ Manuel Ticona Rendón ${ }^{2 b}$ Rubens Pérez Mamani ${ }^{3 \mathrm{c}}$}

Investigador independiente. Tacna, Perú.

2. Escuela Profesional de Medicina Humana de la Universidad Nacional Jorge Basadre. Tacna, Perú.

Escuela de Posgrado de la Universidad Nacional Jorge Basadre Grohmann. Tacna, Perú.

${ }^{\text {a. }}$ Médico Cirujano.

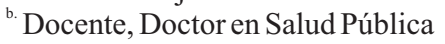

Docente, Ingeniero Comercial.

\section{RESUMEN}

La preeclampsia constituye una de las principales causas de mortalidad materna y perinatal. Objetivo: Determinar los resultados maternos y perinatales de la preeclampsia. Material y métodos: Estudio analítico de casos y controles, se incluyó a todas las madres cuyos partos fueron atendidos en el Hospital Hipólito Unanue de Tacna durante los años 2014 a 2018, los datos se obtuvieron del Sistema Informático Perinatal. Se comparó los casos con los controles mediante frecuencias absolutas y relativas. Se utilizó el estadígrafo Chi2, considerando asociación significativa cuando el " $p$ " sea menor de 0,05 . Para la determinación de resultados maternos y perinatales adversos se utilizó el Odds Ratio con intervalo de confianza al $95 \%$, considerando riesgo significativo cuando el intervalo de confianza esté por encima de 1. Resultados: La incidencia de gestantes con preeclampsia fue de 2,8\%. Los resultados maternos asociados a la preeclampsia fueron: desprendimiento prematuro de placenta $(\mathrm{OR}=6,41)$, terminación por cesárea $(\mathrm{OR}=43,13)$ y muerte materna $(\mathrm{OR}=11,60)$. Los resultados perinatales fueron: prematuridad (OR $=9,63)$, pequeños para la edad gestacional $(\mathrm{OR}=$ $3,80)$, Apgar al primer minuto de 4 a $6(\mathrm{OR}=2,51)$, Apgar a los 5 minutos de 0 a $3(\mathrm{OR}=6,29)$, hipoglicemia $(\mathrm{OR}=2,65)$ y mortalidad perinatal (OR $=3,94)$ en comparación con los recién nacidos de madres sin preeclampsia. Conclusiones: $\mathrm{La}$ preeclampsia se asocia a mayor morbilidad y mortalidad materna y perinatal.

Palabras clave: Factores de riesgo, morbilidad materna, morbilidad neonatal, mortalidad perinatal, Preeclampsia.

\section{ABSTRACT}

Preeclampsia is one of the leading causes of maternal and perinatal mortality. Objective: To determine the maternal and perinatal results of preeclampsia. Material and methods: Analytical study of cases and controls, all mothers whose deliveries were attended at the Hipólito Unanue Hospital in Tacna during the period 2014 to 2018, the data were obtained from the Perinatal Computer System (SIP). Cases were compared with controls using absolute and relative frequencies. Chi2 statistic was used, considering significant association when the "p" is less than 0.05 . For the determination of adverse maternal and perinatal outcomes, the Odds Ratio with $95 \%$ confidence interval was used, considering significant risk when the confidence interval is above 1. Results: The incidence of pregnant women with preeclampsia was $2.8 \%$. . The maternal outcomes associated with preeclampsia were: premature placental abruption $(\mathrm{OR}=6.41)$, cesarean section termination $(\mathrm{OR}=$ $43.13)$ and maternal death $(\mathrm{OR}=11.60)$. Perinatal outcomes were: prematurity $(\mathrm{OR}=9.63)$, small for gestational age $(\mathrm{OR}=3.80)$, Apgar at the first minute of 4 to $6(\mathrm{OR}=2.51)$, Apgar at 5 minutes of 0 at $3(\mathrm{OR}=6.29)$, hypoglycemia $(\mathrm{OR}=2.65)$ and perinatal mortality $(\mathrm{OR}=3.94)$ compared to newborns whose mothers did not present preeclampsia. Conclusion: Preeclampsia is associated with greater maternal and perinatal morbidity and mortality.

Keywords: Risk factors, maternal morbidity, neonatal morbidity, perinatal mortality, preeclampsia 


\section{Introducción}

La preeclampsia es una enfermedad de origen obstétrico que tiene impacto en la salud materna y perinatal, alterando de forma significativa la morbilidad y la mortalidad de la madre, feto y recién nacido. En África y Asia, casi una décima parte de las defunciones maternas están relacionadas con los trastornos hipertensivos, incluida la preeclampsia durante la gestación; mientras que en América Latina, una cuarta parte de las defunciones maternas se relacionan con esas complicaciones(1).

Los trastornos hipertensivos son responsables de la mortalidad materna, ocupando el primer lugar en países desarrollados y siendo la tercera causa de muerte en países en vías de desarrollo como el nuestro(2). En el Perú, estos trastornos representan la segunda causa de muerte materna con $21,3 \%$ de las muertes en el año 2017(3).

Se evidencian alteraciones en el desarrollo del producto, tales como bajo peso al nacer, prematuridad, alteraciones en el Apgar al primer y quinto minuto (4). Siendo un problema en la actualidad, ya que existen complicaciones que pueden comprometer la vida del recién nacido.

A su vez, se evidencia factores de riesgo y consecuencias en las gestantes con preeclampsia que son comunes en esta población. La edad, el grado de instrucción, el estado civil y la culminación del parto (eutócico o cesárea) son, en forma general, el denominador común que presentan las gestantes con preeclampsia(5).

El objetivo de este estudio fue determinar los resultados maternos y perinatales de la preeclampsia en el Hospital Hipólito Unanue de Tacna durante los años 2014 a 2018.

\section{Material y métodos}

Se realizó un estudio analítico, de casos y controles. Se incluyó como casos a todas las gestantes con el diagnóstico de preeclampsia y como controles a todas las gestantes sin preeclampsia, cuyo parto fue atendido en el Hospital Hipólito Unanue de Tacna

La información se obtuvo de la base de datos del Sistema Informático Perinatal, el cual contiene la información de las historias clínicas perinatales. En él se recopila información general de la madre, datos del embarazo, parto y puerperio, además de las complicaciones maternas, características del neonato y complicaciones perinatales. La información fue exportada en el software SPSS versión 23 para el análisis estadístico.
Para el análisis estadístico de las variables cuantitativas, se utilizó estadística descriptiva y medidas de tendencia central como: la media y desviación estándar. Además, para las variables cualitativas solo se utilizó frecuencias absolutas y relativas.

Se presentan tablas comparativas entre los dos grupos (casos y controles), en ambos grupos se comparó las frecuencias absolutas y relativas. Para la determinación de la asociación de variables, se utilizó el estadígrafo Chi2, considerándose asociación significativa cuando el "p" sea menor de 0,05 . Para la determinación de resultados maternos y perinatales adversos se utilizó el Odds Ratio con intervalo de confianza al $95 \%$, considerandose riesgo significativo cuando el intervalo de confianza esté por encima de 1.

\section{Resultados}

En el Hospital Hipólito Unanue de Tacna durante el periodo 2014-2018, se registraron 19671 partos, de los cuales, por los criterios de exclusión, trabajamos con 14756 madres y sus recién nacidos. De dicha población se presentaron 413 casos de preeclampsia $(2,79 \%)$ y nuestro control estuvo representada por una población de 14343 (Tabla 1).

Tabla 1. Incidencia de la preeclampsia en el Hospital Hipólito Unanue de Tacna 2014-2018

\begin{tabular}{lcc} 
Gestantes & $\mathrm{n}$ & $\%$ \\
Con preeclampsia & 413 & $2,79 \%$ \\
Sin preeclampsia & 14343 & $97,20 \%$ \\
Total & 14756 & $100 \%$ \\
\hline
\end{tabular}

En la tabla 2 se observa que de las gestantes con preeclampsia el $25,9 \%$ y $15,6 \%$ en gestantes sin preeclampsia presentaron edad igual o mayor a 35 años. Se encontró que las madres con 35 años a más, tienen 1.84 veces mayor probabilidad de presentar preeclampsia en comparación con las madres de 20 a 34 años, siendo esto un riesgo estadístico significativo. El $71,2 \%$ de las madres con preeclampsia presentaron estado civil de conviviente, el 14,5\% fueron solteras y el $14.3 \%$ fueron casadas, el estado civil no se asoció a esta patología.

Se encontró que el 16,9\% de las mujeres con preeclampsia tuvieron educación superior universitaria; mientras que de las gestantes sin preeclampsia, el 19,8 $\%$ tuvo este nivel de instrucción, presentando 1,75 veces de presentar preeclampsia en comparación con las madres de estudios secundarios. 
Tabla 2. Factores de riesgo socioeconómicos de la preeclampsia Hospital Hipólito Unanue de Tacna 2014-2018

\begin{tabular}{lccccccc}
\multicolumn{7}{c}{ Casos } & \multicolumn{2}{c}{ Controles } \\
& $\mathrm{n}$ & $\%$ & $\mathrm{n}$ & $\%$ & O.R. & IC $95 \%$ & $\mathrm{P}$ \\
\hline Edad $>35$ años & 107 & $25,9 \%$ & 2235 & $15,6 \%$ & 1,84 & $1,46-2,31$ & $<0,001$ \\
$\begin{array}{l}\text { Estudio superior } \\
\text { universitario }\end{array}$ & 70 & $16,9 \%$ & 1542 & $10,8 \%$ & 1,75 & $1,33-2,29$ & $<0,001$
\end{tabular}

En la Tabla 3 se puede observar que el $36,3 \%$ de las madres con preeclampsia presentan sobrepeso, seguido de $34,4 \%$ con obesidad, en los controles presentaron sobrepeso y obesidad $36,3 \%$ y $20,7 \%$ respectivamente. Se encontró que las madres con sobrepeso tienen 1.46 veces mayor probabilidad de tener preeclampsia. Las madres con obesidad presentan mayor riesgo en 2,42 más veces.

Se encontró que el 47,7 \% de las madres con preeclampsia tuvieron controles prenatales adecuados, seguido del 29,8\% con controles prenatales inadecuados y del $22,5 \%$ que no presentaron controles; mientras que de las gestantes sin preeclampsia, el 66,4 $\%$ tuvieron control prenatal adecuado, el 17,2\% tuvieron control inadecuado y el 16,4\% no presentaron ningún control. Se encontró que las madres con controles inadecuados tuvieron 2,41 mayor probabilidad de presentar preeclampsia. Similar situación presentaron las que no tuvieron ningún control prenatal, las cuales tienen 1,9 de riesgo de presentar preeclampsia en comparación con las madres que sí tuvieron control prenatal adecuado, siendo esto un riesgo estadístico significativo.

Se evidencia que las principales comorbilidades maternas asociadas a la preeclampsia fueron la infección del tracto urinario $(\mathrm{OR}=1,31)$, diabetes mellitus $(\mathrm{OR}=8,76)$ y embarazo gemelar $(\mathrm{OR}$ $=3,64)$.

Tabla 3. Factores de riesgo obstetricos de la preeclampsia en el Hospital Hipólito Unanue de Tacna 2014-2018

\begin{tabular}{|c|c|c|c|c|c|c|c|}
\hline & \multicolumn{2}{|c|}{ Casos } & \multicolumn{2}{|c|}{ Controles } & \multirow[b]{2}{*}{ O.R. } & \multirow[b]{2}{*}{ IC 95\% } & \multirow[b]{2}{*}{$\mathrm{P}$} \\
\hline & $\mathrm{n}$ & $\%$ & $\mathrm{n}$ & $\%$ & & & \\
\hline Sobrepeso pregestacional & 150 & $36,3 \%$ & 5213 & $36,3 \%$ & 1,46 & $1,14-1,85$ & 0,003 \\
\hline Obesidad pregestacional & 142 & $34,4 \%$ & 2973 & $20,7 \%$ & 2,42 & $1,88-3,09$ & $<0,001$ \\
\hline Sin control prenatal & 93 & $22,5 \%$ & 2353 & $16,4 \%$ & 1,91 & $1,48-2,45$ & $<0,001$ \\
\hline Control prenatal inadecuado & 123 & $29,8 \%$ & 2469 & $17,2 \%$ & 2,41 & $1,91-3,03$ & $<0,001$ \\
\hline ITU & 184 & $44,55 \%$ & 5457 & $38,04 \%$ & 1,31 & $1,07-1,59$ & 0,007 \\
\hline Diabetes & 4 & $0,97 \%$ & 16 & $0,11 \%$ & 8,76 & $\begin{array}{l}2,91- \\
26,31\end{array}$ & $<0,001$ \\
\hline Embarazo gemelar & 21 & $5,08 \%$ & 208 & $1,45 \%$ & 3,64 & $2,30-5,77$ & $<0,001$ \\
\hline
\end{tabular}

En la Tabla 4 se evidencia que la principal consecuencia asociada a la preeclampsia es el desprendimiento prematuro de placenta, el cual tiene mayor riesgo en 6,41 veces, con un intervalo de $3,35-12,27$, con $p<0,001$. Todos estos resultados presentaron valor significativo.

Se encontró que el 97,8 \% de las madres con preeclampsia concluyeron sus gestaciones en cesárea, mientras que las gestantes sin preeclampsia el $50,7 \%$ terminaron en cesárea. También, se encontró que las madres con preeclampsia tienen una probabilidad de 43,13 veces de que su gestación culmine por cesárea.

Se encontró que hay mayor riesgo $(11,60)$ de mortalidad materna en las mujeres que presentaron preeclampsia en comparación a las mujeres sanas, siendo esto un riesgo estadístico significativo (Tabla 4). 
Tabla 4. Resultados maternos de la preeclampsia en el Hospital Hipólito Unanue de Tacna 2014-2018

\begin{tabular}{lccccccc}
\multicolumn{7}{c}{ Casos } & \multicolumn{2}{c}{ Controles } \\
& $\mathrm{n}$ & $\%$ & $\mathrm{n}$ & $\%$ & O.R. & IC 95 \% & $\mathrm{P}$ \\
$\begin{array}{l}\text { Desprendimiento } \\
\text { prematuro placenta }\end{array}$ & 11 & $2,66 \%$ & 61 & $0,42 \%$ & 6,41 & $3,35-12,27$ & $<0,001$ \\
Cesárea & 404 & $97,8 \%$ & 7272 & $50,7 \%$ & 43,13 & $22,52-84,56$ & $<0,001$ \\
Mortalidad materna & 1 & $0,24 \%$ & 3 & $0,02 \%$ & 11,60 & $1,20-111,77$ & 0,03
\end{tabular}

En la Tabla 5 se puede observar que los recién nacidos de madres con preeclampsia tienen mayor riesgo de presentar afecciones, tales como ser prematuros $(\mathrm{OR}=9,63)$, pequeños para la edad gestacional $(\mathrm{OR}=3,80)$, Apgar al primer minuto de vida de 4 a $6(\mathrm{OR}=2,51)$, Apgar al quinto minuto de 0 a 3 $(\mathrm{OR}=6,29)$, hipoglicemia $(\mathrm{OR}=2,65)$ y mortalidad perinatal $(\mathrm{OR}=3,94)$ en comparación con los recién nacidos de madres sin preeclampsia, siendo esto un riesgo estadístico significativo.

Tabla 5. Resultados perinatales de la preeclampsia en el Hospital Hipólito Unanue de Tacna 2014-2018

\begin{tabular}{lcccccccc}
\multicolumn{1}{c}{ Resultados perinatales } & \multicolumn{2}{c}{ Casos } & \multicolumn{2}{c}{ Controles } & & & \\
& $\mathrm{n}$ & $\%$ & $\mathrm{n}$ & $\%$ & O.R. & IC 95\% & P \\
Rn prematuro & 167 & $38,48 \%$ & 888 & $6,10 \%$ & 9,63 & $7,85-11,83$ & $<0,001$ \\
Pequeño para edad gestacional & 41 & $9,45 \%$ & 369 & $2,54 \%$ & 3,80 & $2,70-5,34$ & $<0,001$ \\
Apgar al minuto 4-6 & 30 & $6,3 \%$ & 420 & $2,9 \%$ & 2,51 & $1,71-3,68$ & $<0,001$ \\
Apgar a los 5 minutos 0 a 3 & 3 & $0,7 \%$ & 27 & $0,2 \%$ & 6,29 & $2,41-16,41$ & 0,001 \\
Hipoglicemia & 14 & $3,23 \%$ & 181 & $1,24 \%$ & 2,65 & $1,52-4,60$ & 0,001 \\
Mortalidad perinatal & 14 & $3,23 \%$ & 122 & $0,84 \%$ & 3,94 & $2,25-6,91$ & $<0,001$
\end{tabular}

\section{Discusión}

La preeclampsia sigue siendo una de las principales causas de morbilidad y mortalidad materna y perinatal, a nivel mundial se estima que esta patología afecta del 2 al $8 \%$ de todos los embarazos (6). En el presente estudio durante el periodo del 2014-2018, se trabajó con una población de 14756 gestantes atendidas en el Hospital Hipólito Unanue de Tacna, de las cuales 413 fueron pacientes con preeclampsia, lo que significa que el $2,8 \%$ de toda nuestra población presentó preeclampsia, dato similar fue encontrado en un estudio multinacional realizado por Ábalos et al. en 29 países en África, Asia, América Latina y Medio oriente, donde la incidencia de gestantes con preeclampsia fue de $2,16 \%$ (7). Khader et al. '(8) realizaron un estudio sobre la preeclampsia en Jordania, analizando los factores de riesgo y resultados maternos y neonatales asociados a dicha patología. En el estudio se obtuvo una incidencia de $1,3 \%$. Pacheco et al. (4) desarrollaron un estudio acerca de la repercusión de la preeclampsia y eclampsia en la mujer peruana y su perinato entre los años 2000-2006. En referido estudio se trabajó con 310795 gestantes, y la incidencia de preeclampsia fue de 5,1\%, lo cual está dentro de lo establecido en la literatura. En cuanto a los factores de riesgo, existe una variedad de factores que incrementa la probabilidad de preeclampsia, hay ciertas características propias de la mujer que la predisponen a desarrollar dicha patología, en el presente estudio se evidenció que las mujeres mayores de 35 años tienen mayor predisposición a desarrollar preeclampsia (OR 1,84, IC95 \% 1,46-2,31, p $<0,001)$. Nathan et al. (9), en un estudio de madres y perinato asociado a preeclampsia en Sudáfrica, concluye que las mujeres de 30 años a más tenían 3,5\% mayor riesgo de presentar preeclampsia. Bilano et al. (10), en un estudio en países en vías de desarrollo con 276388 madres y sus productos, encontró una asociación importante entre la edad materna mayor a 30 años y el bajo nivel de educación. Los autores concluyen que esas características sociodemográficas colocan a las personas como candidata idónea a desarrollar preeclampsia. Abalos et al. (7) relatan que las mujeres mayores de 35 años tienen 2 veces mayor riesgo a tener preeclampsia. En Cuba, García(11) señala la misma asociación, concluyendo de que la edad materna mayor a los 35 posee mayor riesgo de desencadenar dicho trastorno hipertensivo. Pacheco (4) concluye que las mujeres mayores de 35 años tuvieron un riesgo de 1,51 veces de sufrir preeclampsia en el Perú. 
Las mujeres que presentaron educación universitaria tuvieron mayor riesgo (OR 1,75, IC95 \% $1,33-2,29, p<0,001)$ en relación a las otras gestantes. Tal característica no es similar al estudio de Milano(10), porque vivimos en un ciudad que cuenta con dos universidades, la realidad que en la que vivimos en cuanto educación es diferente a otras sociedades de menos recursos.

El estado nutricional inadecuado condiciona a que las mujeres tengan mayor riesgo a desarrollar preeclampsia. El tener sobrepeso (OR 1,46, IC95 \% $1,14-1,85, \mathrm{p}=0,003)$ y obesidad (OR 2,42 , IC95 \% $1,88-3,09, \mathrm{p}<0,001)$ son factores de riesgo en la gestante. En un estudio, Khader (10) confirmó que la obesidad fue un factor de riesgo para las gestantes con mencionada patología. Al igual que el Índice de masa corporal mayor a 25 representa un factor de riesgo para la preeclampsia.

El no tener controles prenatales o presentar menos de 6 controles significa mayor riesgo de desarrollar preeclampsia, incluso hay estudios que recomiendan que el mínimo número de controles prenatales es 8 , lo cual serviría de factor protector (10). En cuanto a la morbilidad materna, observamos que las infecciones del tracto urinario (OR: 1,31, IC95 \% 1,07$1,59, \mathrm{p}=0,007$ ), diabetes mellitus (OR: 8,76, IC95 \% 2,91- 26,31, p < 0,001), y el embarazo gemelar (O.R. 3.64 , IC95 \% 2,30 - 5,77, $\mathrm{p}<0,001$ ) son las patologías que se asocian a mujeres que presentan preeclampsia. En otros estudios se observó la presencia de hipertensión crónica, diabetes gestacional, enfermedad cardiaca o renal, pielonefritis o infección de tracto urinario y anemia, los cuales se constituyeron como factores de riesgo significativo (12).

Conde (13) encontró que el embrazo múltiple, hipertensión crónica, y la diabetes mellitus fueron las principales comorbilidades halladas en su estudio. Como consecuencia de la preeclampsia, encontramos que un grupo de nuestras pacientes presentaron desprendimiento prematuro de placenta (OR: 6,41, IC95 \% 3,35-12,27, p <0,001).

En cuanto a la culminación de parto, el 97,8 \% de los partos de las preelampticas fueron por cesáreas de emergencia (OR: 43,13, CI 95 \%: 22,52 - 84,56 y p < $0,001)$, dicho resultado es semejante a lo encontrado por Suarez (8). La cesárea se utilizó con mayor frecuencia entre las pacientes con preeclampsia en el 59,3\%, además representaron 5.5 más probabilidades de presentar parto pre término en el estudio de García (14).
Las madres con preeclampsia tienen mayor riesgo $(9,63)$ de que sus productos sean prematuros para la edad gestacional en comparación con los recién nacidos de madres sin preeclampsia, con un intervalo de confianza entre 7,85-10,13, con $p<0,001$. Otro estudio en Jordania concluye que la prematuridad estuvo presente en $30 \%$ de los nacidos de mujeres con preeclampsia, al igual que el bajo peso '(8). Los recién nacidos de madres con preeclampsia tuvieron más riesgo $(1,45)$ de nacer con bajo peso, con intervalo de confianza entre $0,99-2,12$, con $p=0,06$. Datos similares se encuentran en un estudio realizado por la OMS, donde el OR fue de 2,32, con un intervalo de confianza $2,16-2,50(10)$.

Los recién nacidos de madres con preeclampsia tuvieron mayor riesgo $(2,51)$ de tener Apgar al primer minuto de 4 a 6 puntos con intervalo de confianza entre $1,71-3,68$ con $p<0,001$, en comparación con los recién nacidos de madres de control. El Apgar al quinto minuto de 4 a 6 puntos presentó 2,19 veces mayor riesgo con intervalo de confianza entre $0,95-5,03$, con valor de $p=0,1$. A su vez, hay probabilidad de tener Apgar de 0 a 3 puntos en 6,29 veces, con un intervalo de confianza entre $2,41-16,41$, con $p<0,001$. Similares resultados fue encontrado por Pacheco(4), donde se encontró Apgar al minuto 5 menor a 7 puntos con $\mathrm{p}<0,000$. En cuanto a la morbilidad neonatal, el retardo del crecimiento intrauterino, y la hipoglicemia estuvieron presentes en los hijos de madres con preeclampsia. Estos niños presentan mayor riesgo de 6 veces a desarrollar retardo en el crecimiento intrauterino con $p<0,001$; mientras que hay mayor probabilidad ( 2,65 veces) de que ellos presenten hipoglicemia con $p<0,001$. Estos datos son estadísticamente significativos.

La mortalidad materna estuvo presente con 1 paciente.

\section{Conclusiones}

El estudio concluye en que las mujeres con preeclampsia tienen 11 veces mayor riesgo de fallecer. Se encontró que hay mayor riesgo de mortalidad perinatal (3,94 veces más) en los hijos de las mujeres que presentaron preeclampsia en comparación a las mujeres sanas, $\mathrm{p}<0,001$.

Se concluye que la prevalencia de la preeclampsia es baja; sin embargo, tiene mayores probabilidades de morbimortalidad materna y perinatal. 


\section{Referencias bibliográficas}

1. Organización Mundial de la Salud. Recomendaciones de la OMS para la prevención y el tratamiento de la preeclampsia y la eclampsia. Ginebra, 2014.

2. American College of Obstetricians and Gynecologists. Hypertension in pregnancy. Women's Health Care Physicians. Washington DC, 2013.

3. Ministerio de Salud. Boletín Epidemiológico del $\mathrm{P}$ e $\mathrm{r}$ ú $\quad$ D i s p o n i b l e e n : http://www.dge.gob.pe/portal/docs/vigilancia/bole tines/2018/26.pdf

4. Pacheco-Romero J, Villacorta A, Del Carpio L, Velásquez É, Acosta O. Repercusión de la preeclampsia/ eclampsia en la mujer peruana y su perinato, 2000-2006. Revista Peruana de Ginecología y Obstetricia 2014; 60(4):27990.

5. Barreto S. Preeclampsia severa, eclampsia y sindrome HELLP: características maternas y resultado neonatal. Unidad de Cuidados Intensivos Maternos. Instituto Materno Perinatal. Lima, Perú 1999-2000. Revista del Hospital Materno Infantil Ramón Sardá 2002; 21(1):17-23.

6. Norma Técnica, Atención integral de la Salud $\mathrm{N}$ e o n a t a 1 . D i s p o n i b 1 e e n : http://bvs.minsa.gob.pe/local/minsa/3281.pdf

7. Abalos E, Cuesta C, Carroli G, Qureshi Z, Widmer M, Vogel JP, etal. Pre-eclampsia, eclampsia and adverse maternal and perinatal outcomes: a secondary analysis of the World Health Organization Multicountry Survey on Maternal and Newborn Health. BJOG: An International Journal of Obstetrics \& Gynaecology. 2014;121(s1):1424.
8. Khader YS, Batieha A, Al-njadat RA, Hijazi SS Preeclampsia in Jordan: incidence, risk factors, and its associated maternal and neonatal outcomes. The Journal of Maternal-Fetal \& Neonatal Medicine. el 19 de marzo de 2018;31(6):7706.

9. Nathan HL, Seed PT, Hezelgrave NL, De Greeff A, Lawley E, Conti-Ramsden F, etal. Maternal and perinatal adverse outcomes in women with preeclampsia cared for at facility-level in South Africa: a prospective cohort study. J Glob Health 2018; $8(2)$.

10. Bilano VL, Ota E, Ganchimeg T, Mori R, Souza JP. Risk Factors of Pre-Eclampsia/Eclampsia and Its Adverse Outcomes in Low- and Middle-Income Countries: A WHO Secondary Analysis. PLOS ONE. el 21 de marzo de 2014; 9(3):e91198.

11. García R, Llera A, Pacheco AL, Delgado M de J, González A. Resultados maternos-perinatales de pacientes con preeclampsia. Revista Cubana de Obstetricia y Ginecología. diciembre de 2012; 38(4):46777.

12. Suárez JA, Veitía M, Gutierrez M, Milián I, López A, Machado F. Condiciones maternas y resultados perinatales en gestantes con riesgo de preeclampsia - eclampsia. Revista Cubana de Obstetricia y Ginecología. marzo de 2017;43(1):1-12.

13. Gómez-Gómez M, Danglot-Banck C. El neonato de madre con preeclampsia-eclampsia.Revista Mexicana de Pediatría2006; 73(2): 82-88.

14. Parra M, San Martín O, Valdés E, Hasbún J, Quiroz L, Schepeler M, et al. Espectro clínico de la preeclampsia: estudio comparativo de sus diversos grados de severidad.Revista chilena de obstetricia y ginecología 2007;72(3): 169-175.

\section{Correspondencia}

angelafzaga@gmail.com
Fecha de recepción: 20 de julio de 2019

Fecha de aceptación: 22 de noviembre de 2019 